\title{
THE IDENTIFICATION OF AN INCOMPLETELY PARTITIONED NETWORK*
}

\author{
By PETER V. O'NEIL (University of Minnesota) and \\ PAUL SLEPIAN (Rensselaer Polytechnic Institute and Sandia Corporation) $\dagger$
}

In [1], the authors presented a formula for the number of trees in a class of networks defined as follows. A network with set of vertices $V$ is incompletely partitioned if, for some positive integer $n$, there exist $2 n$ non empty, pairwise disjoint subsets $A_{i}$ and $D_{i}$ $(i=1,2, \cdots, n)$ of $V$ such that, if $x$ and $y$ are two distinct vertices, then the branch connecting $x$ and $y$ is in the network if and only if there is no $i(i=1,2, \cdots, n)$ such that $x$ is in $A_{i}$ and $y$ in $D_{i}$.

If the network has $m$ vertices, and if, for $i=1,2, \cdots, n, A_{i}$ has $b_{i}$ elements and $D_{i}$ has $c_{i}$ elements, then the number of trees in the network is

$$
m^{m+n-k-2} \prod_{i=1}^{n}\left(m-b_{i}\right)^{c_{i}-1}\left(m-c_{j}\right)^{b_{i}-1}\left(m-b_{i}-c_{i}\right),
$$

where $k=\sum_{i=1}^{n}\left(b_{i}+c_{i}\right)$. Special cases of this formula appear in [2] and [3].

In applying the formula, it is necessary to be able to determine whether a given network is incompletely partitioned, and, if so, to determine the sets $A_{i}$ and $D_{i}$. This can be done as follows.

A bipartite network is said to be fully connected if it includes all possible branches. The following theorem can be proved from the definition in a straightforward manner. If $G$ is an $m$ vertex network, and $G_{m}$ the complete $m$ vertex network, then $G$ is incompletely partitioned if and only if each component of $G_{m}-G$ is a fully connected bipartite network.

The number $n$ appearing in the definition of an incompletely partitioned network is the number of components of $G_{m}-G$, and, for $i=1,2, \cdots, n, A_{i}$ and $D_{i}$ form the partition of the set of vertices of the $i$ th component of $G_{m}-G$ prescribed by the definition of a bipartite network.

\section{REFERENCES}

[1] P. V. O'Neil, and P. Slepian, The number of trees in a network, Proc. Third Allerton Conference on Circuit and System Theory, October, 1965

[2] L. Weinberg, Number of trees in a graph, Proc. I.R.E., Vol. 46, No. 12, Dec. 1958

[3] S. Bedrosian, Formulas for the number of trees in a network, IRE Transactions in Circuit Theory, Vol. PGCT 8, No. 3, Sept. 1961

*Received October 25, 1965; revised manuscript received February 7, 1966.

tThe work of this author was partially supported by the United States Atomic Energy Commission. 